\title{
Les marques de causalité : Etude contrastive des emplois de parce que et comme à partir de données orales (ESLO)
}

\author{
Christophe Mitchito Darmon* \\ Université Kwansei Gakuin, Japon \\ Université d'Orléans, Laboratoire Ligérien de linguistique (UMR-CRNS 7270), France
}

\begin{abstract}
Résumé. De nombreuses études portant sur la causalité se sont intéressées à parce que, car et puisque. Cependant, comme à valeur causale mérite une plus grande attention, car sa structure syntaxique se démarque des autres connecteurs en introduisant la cause avant la conséquence. Cette particularité pourrait-elle induire une perspective différente du rapport de causalité ? En s'appuyant sur le corpus oral ESLO (Enquêtes SocioLinguistiques à Orléans), cet article présente une étude contrastive de comme et parce que qui met en évidence leurs distributions quantitative, syntaxique et discursive à l'oral. Les résultats montrent que parce que peut être dépendant et indépendant syntaxiquement et pragmatiquement. Il peut assurer différentes relations discursives (Explication, Résultat, Arrière-plan), mais les locuteurs l'emploient essentiellement pour exprimer la première. En revanche, comme est moins fréquent, mais révèle une plus grande variété d'emplois discursifs dans le corpus, malgré des contraintes linguistiques plus importantes que parce que. Les tests de substitution le démontrent. Pour finir, les cas litigieux de relations causales et d'arrière-plan avec comme ont révélé une propension à introduire une cause cadrative alors que parce que indique plutôt l'origine de la conséquence. Ainsi chaque connecteur confère intrinsèquement une perspective différente du rapport de causalité.
\end{abstract}

\begin{abstract}
Markers of causality. A contrastive study of parce que and comme based on oral data (ESLO). A large number of studies on causality in French have been dedicated to the connectors parce que, car and puisque. However, comme as a causal connector needs more attention, since its syntactic structure differs from the others by introducing the cause before the consequence. Could this particularity infer different perspectives on the causation relationship? Using the oral corpus ESLO (Enquêtes SocioLinguitiques à Orléans), this article presents a contrastive study of comme and parce que that highlights their quantitative, syntactic, and discursive distribution in speaking. The results show that parce que can be syntactically and pragmatically dependent and independent. It can provide different discursive relationships (Explanation, Result, Background), but speakers mainly use it to express the former. On the other hand, comme is less frequent in the corpus but reveals a greater variety of discursive uses despite greater linguistic constraints than parce que. The substitution tests prove it. Finally, the litigious cases of causal and background relationships with comme have revealed a propensity to introduce a causal framework whereas
\end{abstract}

\footnotetext{
*Corresponding author : mitchitods@gmail.com
} 
parce que indicates the origin of the consequence. Thus, the connectors intrinsically confer a different perspective on the causal relationship.

\section{Introduction}

Les recherches sur la causalité sont nombreuses. Pourtant, si l'opérateur-connecteur parce que a fait l'objet de multiples études, comme reste encore « le parent pauvre » dans ce domaine, comme le souligne Desmets (1998: 1). En effet, les études se sont particulièrement concentrées sur les connecteurs de justification et d'explication parce que, car et puisque. Traditionnellement, nous distinguons d'une part les conjonctions de subordination que sont parce que et puisque et d'autre part la conjonction de coordination car. Les deux premiers sont considérés comme syntaxiquement dépendants et le troisième comme indépendant. Cependant leurs emploies diffèrent selon le rapport causal qu'ils établissent. Tout d'abord, car et puisque sont différents parce que, à la différence du second, le premier ne peut introduire d'arguments déjà connus. Par ailleurs, parce que est surtout employé pour créer des liens causaux plutôt qu'argumentatifs, mais ses emplois sont beaucoup plus variés du fait de sa propension à remplacer car à l'oral. Wüest (2012 : 11) a montré que les emplois de parce que relèvent le plus souvent de la causalité, plus rarement de l'argumentation. En effet, les relations cause-conséquence « sont considérées par le sujet parlant comme étant incontestables et acceptées comme telles par l'allocutaire », l'argumentation étant moins assurée, elle « sert à rendre plus acceptable un acte de langage, dont l'acceptabilité n'est pas hors de doute ». Comme le montre Moeschler (2003 : 40), en deçà de cette distinction, il y a l'établissement d'un lien de causalité. Il relève d'une même chaîne causale dans le premier cas et, dans le second, il requiert l'application d'une règle causale comme l'illustre l'exemple 2. Quant à comme, il a dans ce type d'emplois deux valeurs cardinales, l'une est comparative et l'autre causale. Cet article s'intéressera exclusivement à la seconde. Elle a la particularité d'introduire une proposition subordonnée causale antécédente à la proposition principale. Cependant, l'oral pourrait révéler des emplois novateurs qui mettraient en évidence des caractéristiques méconnues.

Ainsi, cette étude vise à clarifier les différences entre comme et parce que à l'oral afin d'en faire une description explicitant leurs nuances discursives. Au nombre de ces différences, le fait que les deux connecteurs ne positionnent pas la cause de la même façon par rapport à la conséquence, antéposée dans un cas et postposée dans l'autre. Cette différence induirait-elle une perspective différente concernant le rapport de causalité ?

Nous répondrons à cette question en commençant par rappeler les caractéristiques des deux connecteurs en question. Puis nous présenterons les théories sur lesquelles nous nous appuyons pour ensuite procéder à l'analyse des données prélevées dans le corpus ESLO2. ESLO (Enquêtes SocioLinguistiques à Orléans) est un projet mené par le Laboratoire Ligérien de Linguistique (LLL) de l'Université d'Orléans. Il est composé de deux corpus réalisés durant deux périodes bien distinctes : ESLO1 entre 1969 et 1974 et ESLO2 depuis 2008. Les deux corpus sont en libre accès sur le site internet http://eslo.huma-num.fr/index.php

Enfin, nous procéderons à l'examen des occurrences relevées et appliquerons des tests paradigmatiques afin de vérifier leur permutabilité. Enfin nous commenterons les résultats.

\section{Les caractéristiques syntaxiques de parce que et comme}

Nous allons commencer par proposer des exemples tirés de notre corpus afin de mettre en lumière les fonctions syntaxico-pragmatiques que parce que peut assurer.

Dans le cas suivant, nous avons affaire à une proposition subordonnée introduite par parce que. Elle est considérée comme dépendante syntaxiquement (groupe $\lambda-1$ et microsyntaxe (Debaisieux 2013)). 
1. Exemple tiré du corpus ESLO2_ENT_1001

BV1: ça fait un peu trop égocentrique parce que on parle un peu que de soi et

Il peut aussi être indépendant syntaxiquement, mais dépendant discursivement (analyse en macrosyntaxe (Debaisieux (2013), Deulofeu (2016)). Ici, il s'agit de l'introduction d'un argument, suivant la perspective selon laquelle l'argumentation se définit comme la liaison de deux chaînes causales par une règle causale (Moeschler 2003 : 39-40). En (2), nous avons ce que Wüest (2012 : 17) appelle l'argumentation par exemplification.

2. Exemple tiré du corpus ESLO2_ENT_1001

BV1: non non c'est des trucs d'ici

BV1: parce que même y a des gamins dehors euh à huit ans ils disent ça et $\mathrm{j}$ 'ai jamais entendu ça chez moi

Chaîne causale 1 : C'est des trucs [une expression] d'ici, parce que ce n'est utilisé qu'ici.

Chaîne causale 2: Il y a des gamins qui disent ça ici et il a jamais entendu ça chez lui (ailleurs) Règle causale : Si les enfants d'ici l'utilisent, mais que les adultes d'ailleurs ne l'utilisent pas, alors cette expression est limitée à ici.

Dans cet exemple, «même », inséré entre la proposition subordonnée causale et la conjonction, est syntaxiquement isolé. En revanche, sa fonction discursive d'explication de l'opinion du locuteur «c'est des trucs d'ici » est conservée. Parce que peut donc servir à introduire une cause syntaxiquement indépendante, mais discursivement dépendante.

En (3), nous rencontrons un cas où parce que permet d'introduire l'explication d'un acte illocutoire en l'espèce une interrogation: (Le double soulignement indique l'acte illocutoire et le simple l'explication).

3. Exemple tiré du corpus ESLO2_ENT_1001

ch_OB1: euh la médiathèque

ch_OB1: non?

ch_OB1: $\underline{\underline{\text { non? }}}$

ch_OB1: pour les b-

$\mathrm{BV} 1$ : jamais mis les pieds non

ch_OB1: non parce que y a y a des DVD et CD des bouquins

Parce que est relié à une question marquée par les points d'interrogation « non ? non ? ». Ainsi, la proposition introduite par parce que n'est pas dépendante syntaxiquement d'une proposition principale. Elle est plutôt rattachée à un acte illocutoire qui justifie l'énonciation. Le locuteur n'explique pas un contenu informationnel, mais il dit pourquoi il a posé sa question. La justification introduite par parce que a une fonction pragmatique.

Pour finir, nous présentons en (4) un cas où parce que justifie l'énonciation qui vient d'être produite :

4. Exemple tiré du corpus ESLO2_ENT_1001

BV1: bah téléchargement bof

$\mathrm{BV} 1:$ parce que le $\mathrm{PC}$ il commence à se faire vieux et il a du mal mais

ch_OB1: non

$\mathrm{BV} 1$ : ouais streaming de temps en temps

Dans cet exemple, nous pouvons observer un énoncé où parce que fournit la raison expliquant un énoncé sans verbe. Il n'est donc pas dépendant syntaxiquement, mais sa fonction pragmatique visant à justifier un énoncé est préservée.

Ainsi, les emplois de parce que sont variés à l'oral et les cas présentés ci-dessus sont avérés dans nombre de recherches (groupe $\lambda-1$ (1975), Debaisieux (2013)). C'est pourquoi, dans cet article nous avons plutôt choisi de nous intéresser à comme (à valeur causale), car ses emplois à 
l'oral semblent être plus divers que ce que la grammaire traditionnelle présente et que contrairement à parce que les recherches portant sur ce connecteur restent rares.

Desmets (1998) a néanmoins montré que dans les structures phrastiques, ce comme ne peut se situer qu'en position initiale. Or, nous avons pu relever dans notre corpus des cas inverses où comme est postposé à la conséquence comme on le verra en (5). L'intonation suspendue à la fin du segment confirme qu'il s'agit d'un comme causal, même si dans l'absolu il est possible de l'interpréter comme une comparaison. HF8MAR répond à une question lui demandant si le voisinage est calme. Il explique alors qu'il y a des enfants qui crient parce qu'ils jouent au ballon. « comme maintenant y a le ballon » explique pourquoi les enfants poussent des cris. L'exemple montre ainsi qu'à l'oral, l'ordre syntaxique canonique peut s'inverser et que de plus amples analyses sont nécessaires pour déterminer les caractéristiques de comme.

5. Exemple tiré de ESLO2_ENT_1008

HF8MAR: toujours y a les cris toujours y a les

HF8MAR: comme maintenant y a le ballon

Comme dit plus haut, c'est l'intonation qui permet de maintenir le rapport de causalité syntaxiquement. Cependant, le fait d'introduire la cause après la conséquence change la relation discursive entre les deux éléments. En effet, lorsque la cause est antéposée, nous parlons de relation de Résultat alors que quand elle est postposée, celle-ci devient discursivement une Explication. Nous reviendrons sur ces termes dans la partie qui suit.

Parce que a fait l'objet de nombreuses recherches au niveau discursif aussi. Savreux (2017) repère dans ses données une grande variété d'usages discursifs causaux de parce que, notamment celle d'Explication. Cependant il ne parle pas de relation de Résultat. Dès lors que comme peut s'affranchir de la syntaxique, parce que le peut-il ?

Ainsi, la section 4 proposera une analyse de notre corpus afin de savoir si des cas de pragmatisation apparaissent. Cependant, nous commencerons par présenter les travaux de Moeschler $(2003$; 2004 ; 2012) qui explicitent les relations discursives impliquées dans la causalité et nous utiliserons ses théories pour examiner les cas relevés dans notre corpus.

\section{Description de Moeschler sur les relations causales entretenues par les éventualités}

Moeschler (2004) s'applique particulièrement à expliciter les relations causales qui sont impliquées par les emplois de parce que. Pour ce faire il se sert des concepts d'état (dans lequel il regroupe état et activité) et d'événement pour les distinguer, sachant que l'ensemble des états et des événements est désigné par le terme d'éventualité. Dans les exemples les états seront notés « $\mathrm{s}$ » et les événements « e ».

1. les états sont non bornés, homogènes, atéliques et statiques

2. les activités sont non bornées, homogènes, atéliques et dynamiques

3. les événements sont bornés, hétérogènes, téliques et dynamiques, duratifs dans

le cas des accomplissements, ponctuels dans celui des achèvements. Moeschler (2003 : 19)

Il se sert notamment de ces notions pour expliquer les différences d'interprétation causale (au sens strict) et explicative. La causalité directe (au sens strict) implique nécessairement un événement sans discontinuité temporelle, alors que la causalité indirecte (au sens faible), des éventualités sans contigüité temporelle. En voici quatre exemples représentant chacun des cas:

6. Causalité directe $: \mathrm{s}-\mathrm{e}$ (« $\mathrm{s} »$ est souligné et «e » est en italiques)

ch_OB1: non mais c'est marrant parce qu'il me racontait que des fois $i$ -

ch_OB1: quand quand ils faisaient ça ils y allaient à fond et euh 
ch_OB1: euh il retu- il se retrouvait endormi mais il s'est déjà endormi en train de monter une prise euh

7. Causalité directe : e - e

BV1: il essaie de gérer plus les chantiers parce que l'effectif il augmente et

8. Explication : $\mathrm{s}-\mathrm{s}$

BV1: on est $\mathrm{j}$ - on va dire que c'est une boîte de jeunes parce que

BV1: je sais pas le plus âgé Momo il doit avoir euh

BV1: je sais pas je pense que la personne la plus âgée elle doit avoir trente-quatre ans

9. Explication : $\mathrm{e}-\mathrm{s}$

ch_OB1: p- par la suite vous allez conserver le ce ce boulot-là ou euh

$\mathrm{BV} 1$ : bah euh je sais pas pour le moment je me tâte parce que j'ai un projet professionnel

BV1: qui a rien à voir avec ça mais

En (6) et (7), la cause est un événement alors qu'en (8) et (9), c'est un état. Voilà pourquoi Moeschler (2004 : 21) parle de causalité au sens strict dans les deux premiers cas et d'explication dans les seconds. En effet, dans (6), « qu'il me racontait» engendre le fait que " c'est marrant» et dans (7), le fait que "l'effectif augmente » induit « qu'il essaie de gérer plus les chantiers ». En revanche, dans (8), le fait que Momo est la personne la plus âgée et qu'il doit avoir trentequatre ans explique que le locuteur définisse son entreprise comme « une boîte de jeunes ». Quant à (9), le projet professionnel de BV1 explique qu'il hésite (« je me tâte ») à conserver son travail. Parce que peut donc exprimer deux types de causalité en fonction des types d'éventualité (état et événement) qu'il relie. Il peut soit exprimer une causalité directe, soit une explication qui assume les relations de causalité dont la cause est un état.

Ainsi, les éventualités sont solidarisées dans une phrase, un texte, un discours voire un dialogue par des relations de discours déterminées par l'Explication. Il existe de nombreux types de relation de discours qui structurent l'architecture du sens véhiculé dans un ensemble d'éventualités cohérentes.

La relation de Résultat est la forme inverse de l'Explication, car elle relie bien une causalité inter-événementiel, mais la cause vient avant la conséquence. Atallah (2014: 169) la décrit ainsi :

La relation de Résultat, comme celle d'Explication, exprime un lien causal, [...] si ce

n'est l'ordre des arguments du prédicat causal qui change

Atallah (2014 : 169)

A priori, comme mentionné plus haut, si nous nous en tenons à la recherche de Desmets (1998), nous pouvons supposer que comme, qui introduit la cause avant la conséquence, relie uniquement des relations de Résultat. Nous avons trouvé de nombreuses occurrences dans notre corpus. C'est le cas en (10):

\section{Résultat : $\mathrm{s}-\mathrm{e}$}

EH5: comme elle aime l'histoire elle a adoré

Ici, le fait qu' « elle aime l'histoire » est la cause antéposée à la conséquence « elle a adoré ». La relation qui relie les deux éventualités est donc une relation de Résultat.

Toutefois, nous savons aussi que Atallah (2014 : 413) parle de relation d'Explication en ce qui concerne ce connecteur. (5) confirme cette affirmation, également en (11) :

11. Explication : $\mathrm{s}-\mathrm{s}$

VB16: c'est vrai qu'il reste très peu de collègues avec des petits enfants

VB16: comme c'est un centre vieillissant

Dans cet exemple, la cause « c'est un centre vieillissant » est postposée à la conséquence « c'est vrai qu'il reste très peu de collègues avec des petits enfants $»$. 
Comme peut donc aussi bien assumer les relations de Résultat que d'Explication.

\section{Le corpus ESLO}

Nous utiliserons ESLO2 dans la mesure où nous ne cherchons pas à faire une étude diachronique et que nous désirons appuyer nos descriptions sur la langue parlée actuelle. Il est constitué de 18 modules dont le nombre de documents et la durée varient. Nous avons choisi les Entretiens, car c'est le module qui rassemble le plus grand nombre de documents. Par ailleurs, étant donné que les entretiens suivent une trame où les locuteurs témoins expliquent leur trajectoire personnelle et décrivent leurs pratiques situées dans la ville, ce sont les enregistrements qui nous offriront le plus de cas d'Explication et de structures causales. Actuellement, 84 enregistrements sont disponibles sur le site.

La segmentation suit quelques règles qui permettent de définir les sections, les locuteurs et les segments. Les sections distinguent chaque question ou thème de la trame d'entretien. Pour ce qui est des locuteurs, une base de données attribue deux lettres et un chiffre à chacun. Lorsqu'il s'agit d'un chercheur "ch_» et un chiffre lui sont attribués pour toutes les transcriptions où il apparaît. Enfin, la segmentation en unités obéit aux trois règles suivantes :

$>$ les segments ne doivent pas dépasser 15 secondes et dans la mesure du possible ne pas dépasser deux lignes du logiciel de transcription et d'annotation Transcriber ;

$>$ ils représentent des unités syntaxiques et sémantiques cohérentes ;

$>$ ils ne sont pas segmentés mot à mot mais par groupes pertinents.

Nous avons effectué deux requêtes afin d'obtenir les données de notre étude. Tout d'abord, nous avons recherché dans la base de données les occurrences de "parce » dans l'ensemble des entretiens en donnant pour condition d'extraire toutes les occurrences du mot exact. 5545 segments contiennent au moins une occurrence de notre requête sur un total de 1056851 segments disponibles (pour le corpus ESLO2). Sur un total de 23 enregistrements analysés sur 84, il y a 1547 occurrences de parce que.

En ce qui concerne comme, nous avons cherché le mot exact dans la base de données, mais sans spécifier comme critère de choix ceux dont les segments commencent par cet item, car nous souhaitions identifier des cas où un comme à valeur causale se placerait autrement qu'en position initiale. 3526 segments contiennent au moins une occurrence de notre requête sur un total de 1056851 segments disponibles (pour le corpus ESLO2).

Cependant, ce résultat comprend également les comme à valeur comparative. Il fallait donc procéder à la discrimination entre les deux formes de comme manuellement en observant chaque occurrence dans son contexte. Par ailleurs, pour certaines occurrences, la lecture des transcriptions n'était pas suffisante, car seule l'intonation pouvait livrer l'intention du locuteur, comme en (5).

$\mathrm{Au}$ final, sur les 23 enregistrements analysés manuellement, nous avons trouvé 104 occurrences de comme à valeur causale contre 779 occurrences de comme à valeur comparative.

Tableau 1. Total des occurrences de parce que et comme sur 23 entretiens d'ESLO2.

\begin{tabular}{|l|c|c|}
\hline & Parce que & Comme (à valeur causale) \\
\hline $\begin{array}{l}\text { Nombre d'occurrences relevées } \\
\text { dans 23 enregistrements }\end{array}$ & 1547 & 104 \\
\hline
\end{tabular}

Dans notre corpus, les occurrences de parce que sont approximativement 15 fois supérieures à celles de comme. Cette rapide analyse quantitative indique une tendance de l'organisation discursive de la causalité en français. Elle montre que la cause est plus souvent postposée à l'effet. 


\section{Discussion des cas litigieux et définition de l'environnement linguistique de parce que et comme}

Dans cette partie nous étudierons les attributs syntaxiques et discursifs des deux connecteurs.

\subsection{La distribution des temps et des modes autour de parce que et comme}

Tout d'abord, concernant les temps et modes impliqués par parce que et comme, il n'y a pas de différence flagrante. En effet, tous deux relient majoritairement des présents de l'indicatif. Pour parce que, sur 50 occurrences analysées, 29 sont au présent dans la proposition contenant la conséquence et dans celle contenant la cause. En ce qui concerne comme, 32 occurrences sur 84 sont dans le même cas. D'autres configurations ont été relevées comme de l'imparfait pour la cause et du passé composé pour la conséquence.

Ces observations montrent que la majeure partie des temps utilisés dans la proposition introduisant la cause n'en font pas un antécédent temporel de la conséquence, mais plutôt un événement simultané. Dans nos exemples, nous avons repéré seulement 10 occurrences de parce que introduisant une cause qui précède chronologiquement sa conséquence et 14 pour comme. $\mathrm{Ce}$ n'est pas le cas de 40 pour parce que et de 70 pour comme.

12. XF20MER: elle nous aime bien parce que je la fais rire

En (12), la relation de la cause à sa conséquence n'est pas due à l'antériorité temporelle, mais plutôt au fait que XF20MER fasse rire son amie, raison pour laquelle celle-ci les aime bien, son mari et elle.

13. WA7FEM: euh mais mais comme ils parlent aussi bien le français y a pas de y a pas de problème

De même, en (13) avec comme, il n'y a pas de relation d'antériorité-postériorité temporelle du rapport de cause-conséquence. Dans cet énoncé, l'opinion exprimée ( « y a pas de problème ») est soutenue par une circonstance explicative («parlent aussi bien le français »).

Cependant, le présent de l'indicatif n'est pas le seul temps où s'observe une simultanéité temporelle. On rencontre un imparfait décrivant un contexte expliquant l'action de la conséquence au passé composé.

14. EH5: mais euh on a eu la chance de pouvoir y assister parce que l'organisme euh enfin le le

EH5: les scouts invitaient les familles proches donc comme c'était à Chambord

EH5: on a pu y assister

En (14), l'action (au passé composé) est située dans le passé, mais la cause introduite par parce que et celle qui suit comme sont toutes deux à l'imparfait. Les causes énoncent les circonstances expliquant l'action d'y « assister ». Il existe des cas d'antériorité temporelle comme en (15) :

15. DF22: mais comme j'étais partie de chez mes parents

DF22: je ne n'envisageais pas leur demander de me payer la formation

Le plus-que-parfait employé dans la cause («j’étais partie de chez mes parents ») situe bien un événement qui s'est produit avant la conséquence qu'elle explique («je ne n’envisageais pas leur demander de me payer la formation »). 


\subsection{La distribution des relations discursives introduites par parce que et comme}

Cette partie, traitera des relations discursives : Explication, Explication ${ }^{\text {mod }}$, Explication* Résultat, Résultat* ; Arrière-plan et Cadrage. L'indice "mod" désigne les emplois non-régis et nonvéridicibles alors que "*" les usages justifiant un acte illocutoire. Par ailleurs, l'Arrière-plan définit le contexte.et le Cadrage est un de ses sous-types qui donne les informations nécessaires aux inférences permettant à l'interlocuteur d'élaborer une interprétation.

En ce qui concerne la distribution des relations discursives que les deux connecteurs associent, l'analyse a permis d'observer que parce que apparaissait exclusivement dans des cas d'Explication, Explication ${ }^{\bmod }$, Explication* et Arrière-plan alors que comme est employé dans les relations de Résultat et Résultat* en plus de celles introduites par parce que. Cependant, comme privilégie plus particulièrement les relations de Résultat/Résultat* avec 68 occurrences sur 84, 14

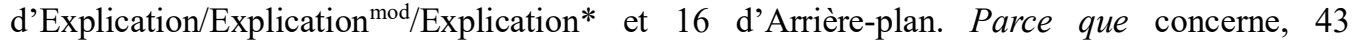

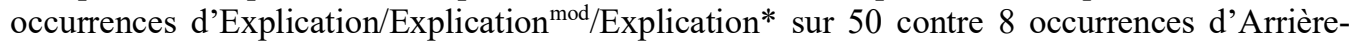
plan. Ces résultats montrent que parce que a la particularité de se placer systématiquement après la conséquence alors que comme peut s'en affranchir.

Précisons que les décomptes ne correspondent pas au total des occurrences relevées, car des cas d'ambiguiité entre Résultat/Explication et Arrière-plan ont été trouvés. Cette question sera traitée en 5.2.2.

\subsubsection{Les relations discursives impliquées par parce que et comme}

Tout d'abord, (16) montre une relation d'Explication qui relie deux événements syntaxiquement dépendants.

\section{Explication}

BV1: il essaie de gérer plus les chantiers parce que l'effectif il augmente et

En revanche, en (17), il s'agit d'une Explication ${ }^{\text {mod }}$ parce que la cause «c'est tranquille c'est autonome » vient appuyer l'acquiescement de BV1 par rapport à l'affirmation non-véridicible de son interlocuteur « pour gagner plus ou pour pour plus d- de liberté euh ».

17. Explication ${ }^{\bmod }$

ch_OB1: ouais ouais pour gagner plus ou pour pour

ch_OB1: plus d- de liberté euh

BV1: bah ouais parce que

BV1: c'est

BV1: enfin d'après les multiples renseignements que je me suis faits euh

BV1: c'est tranquille c'est autonome

Enfin (18), présente une Explication* où parce que a pour fonction de justifier la raison d'un acte illocutoire : poser une question («c'est une idée plutôt récente ça»). C'est l'intonation montante qui montre que c'est une question.

18. Explication*

ch_OB1: c'est une idée plutôt récente ça parce que enfin

ch_OB1: récente

BV1: bah ça fait

BV1: [pf:noise:instantaneous]

BV1: allez-y allez-y

ch_OB1: non non mais c'est juste euh le le m- 
ch_OB1: euh je me demandais avant pour pour bo-pour bosser dans le bâtiment

ch_OB1: c'est venu c'était u- u- une aussi ou

ch_OB1: ça s'est présen-

BV1: oh non non

Comme relie de nombreux Résultats. La relation de Résultat* est en revanche beaucoup plus rare, car le plus souvent la justification d'un acte illocutoire vient après qu'il a été produit. Nous n'avons repéré qu'un seul exemple.

19. Résultat

ZF4: comme j'ai été rue de Limare euh hm

ZF4: j'ai bien connu tous les parents de la rue d'Illiers

Ici, l'exemple est canonique avec la proposition subordonnée causale rattachée syntaxiquement à la principale.

20. Résultat*

WA7: mais comme vous avez vous êtes resté vous êtes depuis le

ch_NS3: [pi:pronounce:instantaneous]

ch_NS3: depuis toute votre enfance donc euh

ch_NS3: bah justement alors je vais vous demander pourquoi vous n'êtes pas parti

L'occurrence ci-dessus est différente, car comme annonce la raison de la question qui suit («pourquoi vous n'êtes pas parti »). C'est donc une relation de Résultat*.

Comme montre par ailleurs des cas d'Explication ${ }^{\text {mod }}$ et d'Explication* respectivement dans les exemples 21 et 22. Dans 21, «bah oui » apporte une appréciation de ce qui précède (« je vais à Paris aussi oui »). L'affirmation est donc non-véridicible et comme introduit une Explication qui la vérifie («j'ai l'occasion d'y aller »).
21. Explication ${ }^{\bmod }$
EW15: mais je vais à Paris aussi oui
EW15: [rire:noise:instantaneous]
ch_AC7: [rire:noise:instantaneous]
EW 15 : bah oui comme $j^{\prime}$ ai l'occasion d'y aller
ch_AC7: c'est l'occasion comme y a les enfants à Paris
EW 15 : oui oui oui

Enfin en 22, comme explique pourquoi ch_BH8 a posé la question « est-ce que ça va pas te poser problème par rapport à ton boulot ».

22. Explication*

ch_BH8: et euh est-ce que ça va pas te poser problème par rapport à ton boulot comme tu me disais que tu travailles plutôt à enfin euh

DF22: j'ai dix minutes de plus de trajet en gros

\subsubsection{Les ambiguïtés avec les relations d'Arrière-plan}

Les relations énumérées jusqu'ici ne sont pas les seules. A l'instar d'Atallah (2014: 413) et Savreux (2017 : 167-180), nous avons aussi repéré des cas où les connecteurs établissent une relation d'Arrière-plan. Celle-ci se définit comme une relation définissant le contexte (Tanguy 2009 : 95). C'est le cas en (23) avec parce que et en (24) avec comme. 


\section{Arrière-plan}

RL2: ils m'ont dit écoutez euh

RL2: on peut pas vraiment vous proposer une formation parce que euh

RL2: bah j'avais pas réellement le niveau à part un CAP pour eux

RL2: ça correspondait à rien

ch_CD2: ouais hm

RL2: euh

RL2: allez faire euh une préformation ils avaient appelé ça allez faire un stage allez

En (23), parce que permet d'insérer un élément d'Arrière-plan à la première personne du singulier dans un discours rapporté à la deuxième personne du pluriel. Et en (24), l'Arrière-plan s'insère dans la proposition principale elle-même.

\section{Arrière-plan \\ UC12: moi c'est quelque chose qui m'intéressait depuis un moment \\ $\mathrm{UC12}$ : comme je travaille en plus qu'à mi-temps euh \\ UC12: de travailler dans l'évènementiel d'organiser ce genre de trucs ouais \\ ch PP6: d'accord \\ $\mathrm{UC} 12$ : ça me motivait bien}

Si ces deux exemples sont sans conteste des relations d'Arrière-plan, pour certains, il est difficile de trancher entre un Arrière-plan et une Explication/Résultat.

\section{Ambiguïté Résultat - Arrière-plan}

SD14: donc lui je le connaissais pas sa femme comme elle vient du sud ouest je la connaissais pas évidemment

Dans cet exemple, nous ne pouvons pas savoir si « elle vient du sud ouest » est la cause de « je la connaissais pas évidemment » ou bien s'il s'agit d'une information d'Arrière-plan qui apporte une information annexe sur «sa femme ». Cela s'infère de la dépendance syntaxique à la principale de la proposition introduite par comme. Ainsi, on peut s'interroger sur la valeur de l'Arrière-plan, car dans le cas de la causalité, le cadre contextuel peut apporter les informations expliquant une conséquence. S'il ne peut avoir de valeur causale au sens strict du terme, du fait que, par définition, il n'est pas le déclencheur de la conséquence, il peut toutefois introduire une valeur explicative, car il donne des indices pour comprendre la conséquence.

Sur les 16 relations d'Arrière-plan que nous avons repérées dans les occurrences de comme, 14 révèlent une ambiguïté entre la relation causale d'Explication/Résultat et celle d'Arrière-plan ; une seule avec parce que, sur 8 occurrences. Selon nous, cette particularité montre que parce que focalise sur le rapport explicatif tandis que comme insiste sur le contexte explicatif. C'est d'ailleurs pour créer cet effet cadratif qu'il est possible de rencontrer des cas où parce que ne suffisant pas, il est combiné à comme :

Tableau 2. Test de suppression de comme.

\begin{tabular}{|l|l|l|}
\hline 26. & \multicolumn{1}{|c|}{ Texte original } & \multicolumn{1}{c|}{ Test de suppression } \\
\hline JW992: & bah si il cuit encore ton & bah si il cuit encore ton \\
\hline JW992: & ton steak toi parce que & ton steak toi parce que \\
\hline JW992: & comme tu l'aimes à point & tu l'aimes à point \\
\hline
\end{tabular}


La comparaison effectuée en (26), montre la différence d'organisation causale. Le test révèle une cause qui déclenche la conséquence alors que le texte original connote une condition ou une circonstance expliquant la conséquence «il cuit encore ton steak ». Parce que paraît donc impliquer un sens causal plus fort que comme. Autrement dit, parce que est plus adapté pour indiquer une cause déclenchant une conséquence alors que comme met en place un «cadre causal » favorisant l'apparition de la conséquence.

\section{Application du test paradigmatique et analyse des relations de discours de parce que et comme}

$\mathrm{Au}$ vu des résultats quantitatifs obtenus à partir de notre corpus, nous pourrions supposer que parce que et comme sont interchangeables, car ils ont des propriétés très similaires. Tous deux relient majoritairement des propositions au présent de l'indicatif, et leurs emplois en causalité temporelle ne sont pas les plus fréquents. Par ailleurs ils relient des relations d'Explication, Explication $^{\text {mod }}$, Explication* et Arrière-plan. Remarquons néanmoins que comme est le seul à apparaître dans des relations de Résultat et Résultat* et qu'il est plus enclin à assurer les relations d'Arrière-plan que parce que. Quoi qu'il en soit, ces résultats pourraient laisser penser que comme serait en mesure de se substituer à parce que et parce que à comme (sauf dans les cas de Résultat et Résultat*). Dans l'échantillon des occurrences de comme, toutes les occurrences peuvent être remplacées par parce que même si certaines rendraient l'énoncé moins fluide. Par ailleurs, notre analyse montre que parce que est à même de se substituer à comme dans les cas d'Explication.

Tableau 3. Test de substitution 1.

\begin{tabular}{|l|l|l|}
\hline 27. & \multicolumn{1}{|c|}{ Texte original } & \multicolumn{1}{|c|}{ Test de substitution } \\
\hline ch_AC7: & $\begin{array}{l}\text { c'est l'occasion comme y a les enfants à } \\
\text { Paris }\end{array}$ & $\begin{array}{l}\text { c'est l'occasion parce que } y \text { a les enfants } \\
\text { à Paris }\end{array}$ \\
\hline
\end{tabular}

Cependant, de façon inattendue, il peut aussi remplacer comme dans les cas de Résultat comme en (28).

Tableau 4. Test de substitution 2

\begin{tabular}{|l|l|l|}
\hline 28. & \multicolumn{1}{|c|}{ Texte original } & \multicolumn{1}{|c|}{ Test de substitution } \\
\hline ZF4: & comme on avait vécu & Parce que on avait vécu \\
\hline ZF4: & $\grave{a}$ Paris dans le Cher à Nice et en Creuse & $\grave{a ̀ ~ P a r i s ~ d a n s ~ l e ~ C h e r ~ a ̀ ~ N i c e ~ e t ~ e n ~ C r e u s e ~}$ \\
\hline ZF4: & $\begin{array}{l}\text { on était capable de faire un des } \\
\text { différences }\end{array}$ & $\begin{array}{l}\text { on était capable de faire un des } \\
\text { différences }\end{array}$ \\
\hline
\end{tabular}

Même les occurrences d'Arrière-plan n'ont pas posé de problème. Nous pouvons le constater en (29). 
Tableau 5. Test de substitution 3.

\begin{tabular}{|l|l|l|}
\hline 29. & \multicolumn{1}{|c|}{ Texte original } & \multicolumn{1}{|c|}{ Test de substitution } \\
\hline EH5: & $\begin{array}{l}\text { mais je me suis dit comme } \text { il a jamais } \\
\text { fait de scoutisme ça va lui faire peur et } \\
\text { il m'a dit }\end{array}$ & $\begin{array}{l}\text { mais je me suis dit parce que } \text { il a jamais } \\
\text { fait de scoutisme ça va lui faire peur et il } \\
\text { m'a dit }\end{array}$ \\
\hline EH5: & tu as pas envie qu'on le fasse ? & tu as pas envie qu'on le fasse? \\
\hline
\end{tabular}

En revanche, certains cas ont révélé que l'emploi de parce que pouvait paraître étrange en raison de l'impact qu'il a sur l'enchaînement discursif qui diffère de celui de comme (cf.(30)).

Tableau 6. Test de substitution 4.

\begin{tabular}{|l|l|l|}
\hline 30. & \multicolumn{1}{|c|}{ Texte d'origine } & \multicolumn{1}{c|}{ Test de substitution } \\
\hline NX31: & $\begin{array}{l}\text { donc c'est ce que je lui ai dit comme il } \\
\text { les voit qu'une fois par an voire deux fois } \\
\text { par an c'est pas beaucoup }\end{array}$ & $\begin{array}{l}\text { ? donc c'est ce que je lui ai dit parce que } \\
\text { il les voit qu'une fois par an voire deux } \\
\text { fois par an c'est pas beaucoup }\end{array}$ \\
\hline NX31: & donc c'est beaucoup & donc c'est beaucoup \\
\hline ch_NS3: & hm hm & hm hm \\
\hline NX31: & de téléphone beaucoup d'Internet & de téléphone beaucoup d'Internet \\
\hline
\end{tabular}

Dans cet exemple, le test avec parce que ne parait pas exclu, car en fonction de l'intonation les liens discursifs pourraient être modifiés, mais l'emploi reste étrange dans cette position. En effet, parce que semble privilégier le lien à une conséquence qui le précède. Nous aurions alors « donc c'est ce que je lui ai dit parce que il les voit qu'une fois voire deux fois par an ». Cependant, avec cette configuration, «c'est pas beaucoup », isolé, n'est alors plus l'expression d'une conséquence, mais un simple commentaire sur ce qui précède. Or, dans la version d'origine, comme introduit la cause « il les voit qu'une fois par an voire deux fois par an » pour la rattacher à la conséquence : « c'est pas beaucoup ». Ainsi cet exemple montre que l'organisation discursive peut changer sous l'effet du connecteur.

Si on applique le même test aux occurrences de comme, nous pouvons voir qu'il est aussi en mesure de se substituer à parce que. Toutefois, encore une fois l'effet sur le discours n'est pas vraiment le même selon que l'on utilise parce que ou comme.

Tableau 7. Test de substitution 5 .

\begin{tabular}{|l|l|l|}
\hline 31. & \multicolumn{1}{|c|}{ Texte d'origine } & \multicolumn{1}{|c|}{ Test de substitution } \\
\hline$Z F 4:$ & je je vais euh souvent euh & je je vais euh souvent euh \\
\hline ZF4: & $\begin{array}{l}\text { dans le café euh comment s'appelle ce } \\
\text { café le } ?\end{array}$ & $\begin{array}{l}\text { dans le café euh comment s'appelle ce café } \\
\text { le ? }\end{array}$ \\
\hline$Z F 4:$ & {$[$ bg:noise:instantaneous] } & {$[$ bg:noise:instantaneous] } \\
\hline ZF4: & l'Atelier & l'Atelier \\
\hline$Z F 4:$ & euh rue de Bourgogne & euh rue de Bourgogne \\
\hline ZF4: & parce que Baptiste y joue très souvent & comme Baptiste y joue très souvent \\
\hline
\end{tabular}

L'exemple 31 montre que les deux connecteurs sont interchangeables. Cependant, le mécanisme discursif s'avère légèrement différent. Avec parce que, la cause est directement reliée à la conséquence qui précède alors qu'avec comme, le connecteur introduit une cause dont la conséquence, qui devrait apparaître ensuite, est absente. À défaut, l'élément précédent remplit ce rôle ; c'est la solution la plus cohérente. Toutefois, si (31) semble opératoire, un certain nombre de cas se sont avérés moins concluants. Dans l'ensemble des énoncés que nous avons analysés, nous avons rencontré 12 cas provenant tous du corpus de parce que où le test paradigmatique n'a 
pas fonctionné. (32) et (33) sont deux exemples qui montrent des incompatibilités discursives. Afin de les mettre en évidence, nous allons procéder aux tests ci-dessous.

Tableau 8. Test de substitution 6.

\begin{tabular}{|c|c|c|}
\hline 32. & Texte d'origine & Test de substitution \\
\hline ch_OB1: & $\begin{array}{l}\text { [j'ai l'exemple des étudiants étrangers } \\
\text { qui arrivent] }\end{array}$ & $\begin{array}{l}\text { [j'ai l'exemple des étudiants étrangers qui } \\
\text { arrivent] }\end{array}$ \\
\hline ch_OB1: & $\begin{array}{l}\text { [qui me disent on comp- on comprend } \\
\text { rien] }]^{\mathrm{e} 2} \text { [parce que on nous a expliqué } \\
\text { plein de trucs chez nous] }]^{\mathrm{e} 3}\end{array}$ & $\begin{array}{l}\text { [qui me disent on comp- on comprend } \\
\text { rien }]^{\mathrm{e} 2}[\text { comme on nous a expliqué plein } \\
\text { de trucs chez nous }]^{\mathrm{e} 3}\end{array}$ \\
\hline ch_OB1: & $\begin{array}{l}\text { [en disant qu'en français il y avait la } \\
\text { liaison] }]^{\text {e4 }} \text { [qu'il faut dire euh }\end{array}$ & 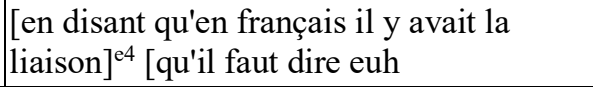 \\
\hline ch_OB1: & euh qui faut dire trois euros et tout $]^{e 5}$ & euh qui faut dire trois euros et tout $]^{\mathrm{e} 5}$ \\
\hline ch_OB1: & $\begin{array}{l}{[\text { et puis euh on va acheter le pain et }} \\
\text { tout }]^{\text {e6 }}\end{array}$ & $\begin{array}{l}{[\text { et puis euh on va acheter le pain et }} \\
\text { tout }]^{\text {e6 }}\end{array}$ \\
\hline ch_OB1: & $\begin{array}{l}\text { [partout tout le monde dit euh deux } \\
\text { euros trois euros euh] }]^{\mathrm{e} 7}\end{array}$ & $\begin{array}{l}\text { [partout tout le monde dit euh deux euros } \\
\text { trois euros euh] }]^{\text {e7 }}\end{array}$ \\
\hline ch_OB1: & [donc euh & [donc euh \\
\hline BV1: & $\mathrm{hm}$ & hm \\
\hline ch_OB1: & $\begin{array}{l}\text { les gens ils prononcent pas comme on a } \\
\text { appris à l'école] }]^{\text {e8 }}\end{array}$ & $\begin{array}{l}\text { les gens ils prononcent pas comme on a } \\
\text { appris à l'école] }]^{\text {e8 }}\end{array}$ \\
\hline ch_OB1: & $\begin{array}{l}\text { bah ouais l- on parle pas comme on a } \\
\text { appris à l'école c'est vrai }\end{array}$ & $\begin{array}{l}\text { bah ouais l- on parle pas comme on a } \\
\text { appris à l'école c'est vrai }\end{array}$ \\
\hline BV1: & ouais bah ouais & ouais bah ouais \\
\hline
\end{tabular}

Tableau 9. Analyse des relations de discours 1.

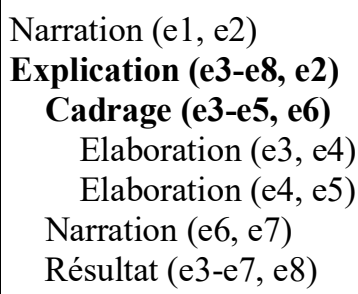

\begin{tabular}{|l} 
Narration (e1, e2) \\
Explication (e3, e2) \\
Cadrage (e3-e5, e6) \\
Elaboration (e3, e4) \\
Elaboration (e4, e5) \\
Narration (e6, e7) \\
Résultat (e3-e7, e8)
\end{tabular}

Dans la version d'origine, nous pouvons voir que parce que signale l'Explication de e2 qui commence à e 3 et finit à e 8 . " on nous a expliqué plein de trucs chez nous » ne pouvant pas être la cause de « qui me disent on comp- on comprend rien », nous interprétons parce que en élément introductif d'un cadrage de l'explication donnée en aval ; le Cadrage étant un type d'Arrièreplan. Or dans le test avec comme, il semble que lorsqu'il se démarque de la structure canonique et qu'il vient après la conséquence, la cause qu'il introduit privilégie la relation d'Explication, mais sans pour autant être en mesure d'introduire un Cadrage. Voilà pourquoi comme ne parvient pas à remplacer parce que dans cet exemple.

En (33), on voit que dans certains cas, comme pourrait accepter un ajout même lorsqu'il est dans une relation d'Explication, mais si celui-ci ouvre vers un développement en Arrière-plan, la structure organisationnelle du discours n'est pas valide. 
Tableau 10. Test de substitution 7 .

\begin{tabular}{|c|c|c|}
\hline 33 & Texte d'origine & Test de substitution \\
\hline ZF4: & $\begin{array}{l}\text { [alors euh par exemple cette se-ce } \\
\text { week-end c'était un week-end hyper } \\
\text { chargé] }]^{\text {e1 }}\end{array}$ & $\begin{array}{l}\text { [alors euh par exemple cette se-ce } \\
\text { week-end c'était un week-end hyper } \\
\text { chargée }]^{\mathrm{el}}\end{array}$ \\
\hline ZF4: & $\begin{array}{l}\text { [parce que mes deux fils euh jouaient } \\
\text { ensemble euh au théâtre euh dans le }\end{array}$ & $\begin{array}{l}\text { [comme mes deux fils euh jouaient } \\
\text { ensemble euh au théâtre euh dans le }\end{array}$ \\
\hline ZF4: & $\begin{array}{l}\text { au jazz l'après-midi à cinq heures } \\
\text { gratos] }\end{array}$ & $\begin{array}{l}\text { au jazz l'après-midi à cinq heures } \\
\text { gratos] }\end{array}$ \\
\hline ZF4: & [et puis euh le soir & [et puis euh le soir \\
\hline ZF4: & $\begin{array}{l}\text { euh mon fils Baptiste accompagnait } \\
\text { Hugo Zermati] }]^{\mathrm{e} 3}\end{array}$ & $\begin{array}{l}\text { euh mon fils Baptiste accompagnait } \\
\text { Hugo Zermati] }]^{\text {e3 }}\end{array}$ \\
\hline ZF4: & [qui est un & [qui est un \\
\hline ZF4: & un & un \\
\hline ZF4: & une sorte de clown magique] ${ }^{\mathrm{e} 4}$ [et & une sorte de clown magique] ${ }^{\mathrm{e} 4}$ [et \\
\hline
\end{tabular}

Tableau 11. Analyse des relations de discours 2 .

\begin{tabular}{|l|c|}
\hline $\begin{array}{c}\text { Explication (e2-e3, e1) } \\
\text { Ajout (e2, e3) } \\
\text { Arrière-plan (e3, e4) }\end{array}$ & $\begin{array}{c}\text { Explication (e2-e3, e1) } \\
\text { Ajout (e2, e3) } \\
\text { *Arrière-plan (e3, e4) }\end{array}$ \\
\hline
\end{tabular}

Dans cet exemple, parce que peut, comme le permet sa forme canonique, donner l'explication après la conséquence tout en étant rattaché à un ajout qui étaie l'explication de la cause : «mes deux fils euh jouaient ensemble euh au théâtre euh dans le au jazz l'après-midi à cinq heures gratos et puis euh le soir euh mon fils Baptiste accompagnait Hugo Zermati » explique en quoi le week-end était « hyper chargé ». Mais parce que permet facilement un développement discursif avec un arrière-plan « qui est un n une sorte de clown magique », ce qui n'est pas le cas de comme. En effet, si comme peut accepter l'ajout en tant que suite explicative de la conséquence antéposée, il ne peut outrepasser plus encore sa forme canonique.

(32) et (33) montrent donc que comme peut bel et bien assurer la relation d'explication à l'oral même si sa forme canonique ne l'autorise pas, mais il ne peut cependant réaliser un développement discursif qui s'écarte au-delà du cadre de l'explication alors que parce que le peut.

\section{Analyse et conclusion}

Tout d'abord, notre étude du corpus ESLO a démontré qu'à l'oral les prescriptions syntaxiques de l'écrit peuvent être transgressées : c'est le cas avec comme dans de nombreux exemples (21, 22,27 , test de substitution en 31) tirés des enregistrements. A l'heure actuelle, nous n'avons pas trouvé d'occurrences attestant ce phénomène avec parce que, mais les tests ont montré qu'a priori l'antéposition de la proposition subordonnée causale ne pose pas de difficulté (tests de substitution en 28 et 29). Cependant, ce choix est sans doute rare en raison de la tendance des locuteurs du français à privilégier la postposition de la cause par rapport à la conséquence. C'est ce que l'analyse quantitative des données suggère : il y a quinze fois plus d'occurrences de parce que que de comme.

Par ailleurs, comme est plus contraint que parce que au niveau syntaxico-discursif. En effet, si parce que est en mesure de remplacer comme dans toutes ses occurrences, à l'inverse comme n'en est pas toujours susceptible alors qu'il démontre une plus grande variété de relations discursives dans le corpus. Nous avons surtout pu voir que dans les cas où parce que introduit un 
paragraphe explicatif impliquant un enchaînement discursif, comme ne peut être extrapolé audelà d'une seule relation d'Explication. Ainsi, (32) et (33) montrent qu'une transgression syntaxique est effectivement envisageable par rapport à la forme canonique de l'emploi du connecteur, mais que pour conserver le lien de cause à conséquence, la chaîne discursive ne peut pas aller au-delà d'un énoncé explicatif.

En outre, l'étude des cas litigieux mettant en évidence la difficulté de distinguer les relations d'Explication/Résultat et celles d'Arrière-plan, montre que les connecteurs imposent une certaine perspective du rapport de causalité selon leurs caractéristiques propres. C'est ainsi que comme privilégie le cadrage pour induire une déduction de la conséquence alors que parce que indique plus largement l'origine de la conséquence. Voilà pourquoi le test de suppression (26) perd son acception circonstancielle lorsque l'on retire comme, et qu'il ne reste que l'interprétation d'une cause déclenchant un effet.

Tous ces constats conduisent à s'interroger sur les relations de causalité qu'impliquent les connecteurs. En effet, si l'antériorité ou la postériorité de la cause par rapport à la conséquence organisent le discours causal différemment du fait de l'ordonnancement syntaxico-discursif, il semble que les connecteurs eux-mêmes induisent une perspective causale. Il paraît donc nécessaire de définir plus précisément les attributs sémantiques des deux connecteurs pour saisir le point de vue inféré par chacun.

Enfin, comme l'a montré l'analyse quantitative des temps et des modes, la causalité ne semble pas privilégier la relation chronologique de cause à effet. Au contraire, la simultanéité temporelle est la plus fréquente. C'est pourquoi la causalité dépendrait avant tout de la perspective choisie par le locuteur pour énoncer le rapport causal tel qu'il le perçoit et non des événements.

\section{Références bibliographiques}

Atallah, C. (2014). Analyse de relations de discours causales : étude empirique et caractérisation théorique (Thèse de doctorat, Université Toulouse Le Mirail, Toulouse).

Debaisieux, J-M. (éds.) (2013). Analyses linguistiques sur corpus, subordination en français. Paris: Lavoisier.

Desmets, M. (1998). Identification de deux constructions en comme : causalité et comparaison. Linx, 39, 89118.

Deulofeu, J. (2016). La macrosyntaxe comme moyen de tracer la limite entre organisation grammaticale et organisation du discours. Macro-syntax as the Means of Tracing the Frontiers between Grammatical and Discursive Organisation. Modèles Linguistiques, 135-166. https://doi.org/10.4000/ml.2040 $(04 / 04 / 2019)$

Ducrot, O. (1984). Le dire et le dit. Paris : Editions de minuit.

Moeschler, J. (2003). L'expression de la causalité en français. Cahier de linguistique française 25, 11-42.

Moeschler, J. (2004). Dialogue et causalité : force causale, actes de langage et enchaînement. Cahier de linguistique française, 67-85.

Moeschler, J. (2012). Pourquoi le sens est-il structuré ? Une approche vériconditionnelle de la signification linguistique et du sens pragmatique. Nouveaux cahiers de linguistique française 30, 53-75.

Savreux, F. (2017). De la syntaxe à la sémantique de discours : étude en corpus des constructions en parce que (Thèse de doctorat, Université Toulouse Le Mirail, Toulouse). En ligne : https:/tel.archivesouvertes.fr/tel-02108897 (07/07/2019)

Tanguy, N. (2009). Les segments averbaux, unités syntaxiques de l'oral (Thèse de doctorat, Université de la Sorbonne nouvelle - Paris 3).

Wüest, J. (2012). Argumentation et causalité : à propos des connecteurs parce que, car et puisque. Vox Romanica $71,77-108$.

Corpus ESLO http://eslo.huma-num.fr/index.php 